\title{
Deletion of the ntrYX Two Component System in Rhodobacter sphaeroides Causes the Generation of Diverse Extracellular Membrane Structures
}

\author{
Daniel Parrell, Kimberly Lemmer, Timothy Donohue and Elizabeth Wright \\ Universit of Wisconsin-Madison, Madison, Wisconsin, United States
}

Rhodobacter sphaeroides is a facultative photoheterotrophic bacterium that is an important model for research into biofuels and the production of primary materials for industrial purposes. In previous work, a genetic screen using a Tn5 transposon insertion library and a Nile red assay for lipid production, $R$. sphaeroides isolates which over produce extracellular lipids were isolated under aerobic growth conditions (1). Determining the mechanisms that lead to the overproduction of lipid secretions in these $R$. sphaeroides isolates may have a broad impact on the production of renewable fuels and chemicals from a biological source. Ten of the highest extracellular lipid producers were analyzed for the loci disrupted through transposon insertion. One of these strains, the highest producer of extracellular lipids, carried an insertion into the ntrYX gene locus. NtrY and NtrX comprise elements of a two-component regulatory system that is known to control exopolysaccharide production and processes controlled by respiration or anaerobic growth conditions (2-4).

$R$. sphaeroides cultures were grown at $30^{\circ} \mathrm{C}$ and 200 RPM shaking in Sistron minimal medium until the culture reached an OD600 of 0.6. Suspensions of the cells were then deposited onto 200 mesh R2/1 copper Quantifoil grids in $5 \mu \mathrm{L}$ aliquots, blotted, and plunge frozen in liquid ethane using a Vitrobot Mark IV (Thermo Fisher). Cryo-electron microscopy (cryo-EM) and cryo-electron tomography (cryo-ET) data were collected using either a Titan Krios TEM (ThermoFisher) operated at $300 \mathrm{kV}$, equipped with a Gatan Bioquantum energy filter and a K3 direct electron detector (Gatan) or a Tecnai TF30 TEM (ThermoFisher) operated at $300 \mathrm{kV}$ equipped with a Gatan energy filter and a K2 direct electron detector (Gatan). Single axis tilt series were acquired using SerialEM (5), with an increment of $2^{\circ}$ covering $-60^{\circ}$ to $+60^{\circ}$ and a cumulative dose under $150 \mathrm{e}-/ \AA 2$ at a defocus range between -4 and $-10 \mu \mathrm{m}$. Tomograms were reconstructed using IMOD/eTomo (6), and segmentation was performed using the Amira software package (Thermo fisher).

Cryo-EM and cryo-ET were used to determine whether the deletion of ntrYX in R. sphaeroides compromises cell division and envelope stability. In addition to these phenotypes caused by ntrYX deletion, an increased production of extracellular lipids was also observed as the production of extracellular membrane structures and vesicles (Fig. 1). These observations correlate with structures previously recovered from concentrated culture supernatants after separating cells from the culture medium by centrifugation (1). Our observations show that these structures are closely associated with cells and that their production occurs at the cell surface, consistent with observations that ntrYX disruption causes instability to the bacterial envelope. Future work will be to determine the molecular mechanisms that result in cell division defects and instability of the envelope. By developing an improved understanding of the mechanisms by which these $R$. sphaeroides strains overproduce cellular lipids, great strides may be made toward creating better biofuels and chemicals for industrial purposes [7]. 


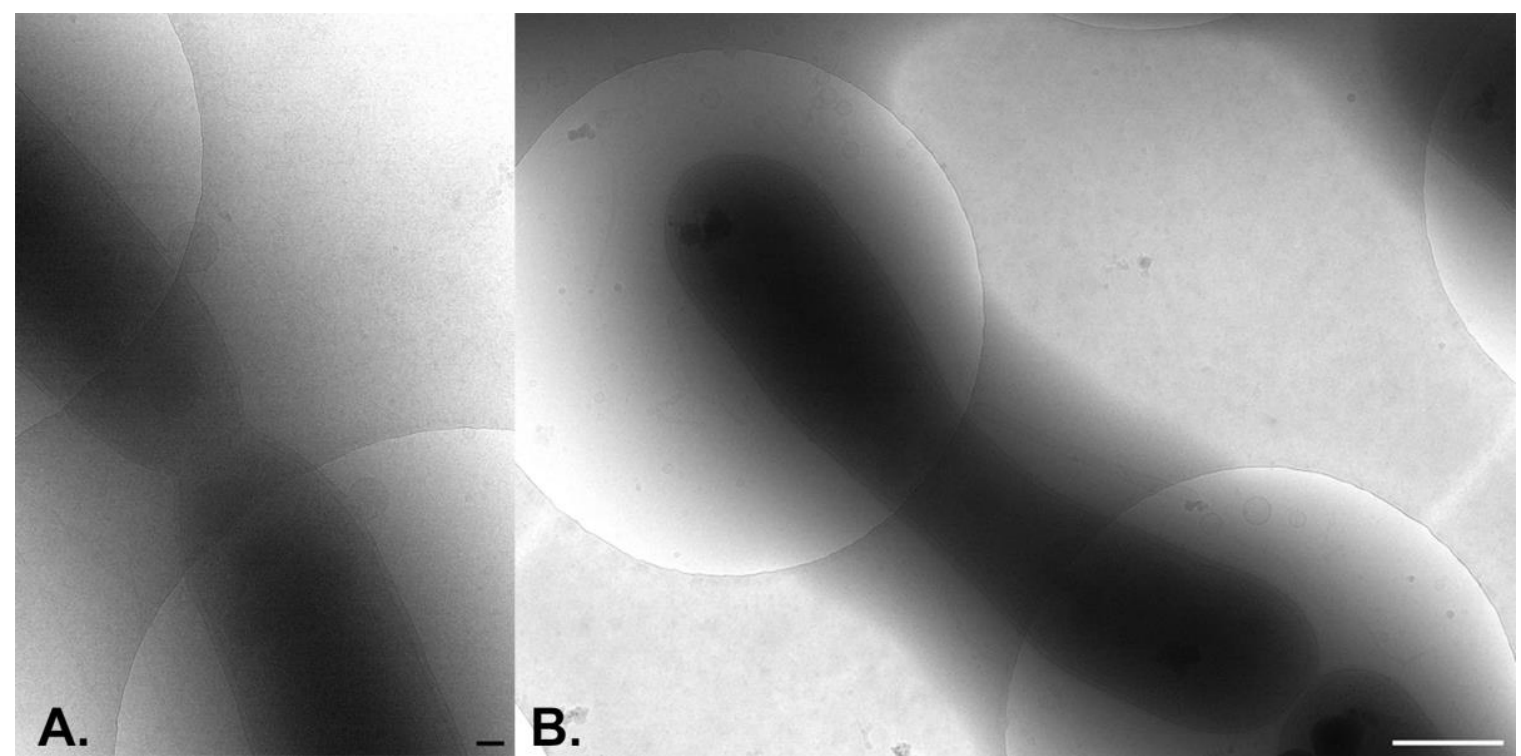

Figure 1. Cryo-EM images of Rhodobacter sphaeroides cells. A) An R. sphaeroides cell producing membrane vesicles by budding off material from the outer cell membrane. The scale bar represents 100 $\mathrm{nm}$. B) An R. sphaeroides cell that has produced membrane vesicles of several sizes, chains of vesicle and a long membrane tubule. The scale bar represents $500 \mathrm{~nm}$.

\section{References}

1. Lemmer KC, Zhang W, Langer SJ, Dohnalkova AC, Hu D, Lemke RA, Piotrowski JS, Orr G, Noguera DR, Donohue TJ. Mutations That Alter the Bacterial Cell Envelope Increase Lipid Production. mBio. 2017;8(3).

2. Wang D, Xue H, Wang Y, Yin R, Xie F, Luo L. The Sinorhizobium meliloti ntrX gene is involved in succinoglycan production, motility, and symbiotic nodulation on alfalfa. Appl Environ Microbiol. 2013;79(23):7150-9.

3. Pawlowski K, Klosse U, de Bruijn FJ. Characterization of a novel Azorhizobium caulinodans ORS571 two-component regulatory system, NtrY/NtrX, involved in nitrogen fixation and metabolism. Mol Gen Genet. 1991;231(1):124-38.

4. Carrica Mdel C, Fernandez I, Sieira R, Paris G, Goldbaum FA. The two-component systems PrrBA and NtrYX co-ordinately regulate the adaptation of Brucella abortus to an oxygen-limited environment. Mol Microbiol. 2013;88(2):222-33.

5. Mastronarde DN. Automated electron microscope tomography using robust prediction of specimen movements. J Struct Biol. 2005;152(1):36-51.

6. Kremer JR, Mastronarde DN, McIntosh JR. Computer visualization of three-dimensional image data using IMOD. J Struct Biol. 1996;116(1):71-6.

7. This work was supported in part by funds from the University of Wisconsin, Madison and the Great Lakes Bioenergy Research Center, U.S. Department of Energy, Office of Sciences, Office of Biological and Environmental Research under award numbers DE-SC0018409 and DE-FC02-07ER64494. The authors gratefully acknowledge use of facilities and instrumentation at the UW-Madison Wisconsin Centers for Nanoscale Technology (wcnt.wisc.edu) partially supported by the NSF through the University of Wisconsin Materials Research Science and Engineering Center (DMR-1720415). 